\title{
SINGLE MOTOR TWO-AXEL SOLAR PANELS TRACKING DEVICE USING SLIDER-CRANK MECHANISM
}

Viktorija Zagorska, Andrejs Snegovs, Henriks Putans, Imants Ziemelis

Latvia University of Life Sciences and Technologies, Latvia

viktorija.zagorska@1lu.lv, andrejs.snegovs@gmail.com, henriksooo@inbox.lv, imants.ziemelis@1lu.lv

\begin{abstract}
The aim of the research was to work out the methodology for the calculation of the accuracy of the created solar panels tracking the sun stand, and perform its experimental verification. The operation of the solar panels is considerably more effective, if their working surface is oriented perpendicularly to the striking sun beams. For that purpose sun tracking stands are used, ensuring the panels face surface oriented perpendicularly to the sunbeams all the working time. Different solar panels tracking stands are in use. Usually these stands are turning the frame of panels in vertical and horizontal plane around two axes, powered by two electric motors. The solar tracking stand in question has only the one electric motor for turning the panels in horizontal and vertical plane. Continuing the development of one-engine twin-drive systems, in which one motor rotates the solar panels in a horizontal (azimuth) plane, while the panel motion trajectory in the vertical (zenith) plane is formed by a slider-clutch mechanism, is a new approach to solve the problem. One end of the slider is immovably connected to the shaft with the solar panels, but another movably to the connecting rod. The slider is perpendicular to the panels face surface, therefore, it has to be parallel to the sunbeams all the time of operation. The methodology of analytical calculation of the position of the slider depending on the solar beam direction has been worked out. The coherence between panels' position in zenith and azimuth planes depending on the slider angle referred to the horizon has been established. The slider's free end angles referring to the horizon depending on time have been calculated and its trajectory has been established. The difference between the trajectory values calculated and experimentally obtained is insignificant.
\end{abstract}

Keywords: solar panels, sun trackers.

\section{Introduction}

The device in question relates to the use of solar energy to generate electricity or heat, which then can used for various economic purposes. A solar panel or a solar collector with a working surface facing the sun all day long can receive until $50 \%$ more energy than a stationary south - facing analogue surface $[1 ; 2]$. Because of this, in many cases it is more advantageous to use a smaller total number of solar panels (the work surface), but to install them on a tracking the sun stand. This is also important when the installation area is limited. Two telescope stands are known from the point of view of the orientation of the sun in the telescopes. In principle, they too are zenith-azimuthal and equatorial stands. Zenith-azimuthal (Dobson) stand has two horizontal and vertical cutting axles and the equatorial stand has one axis: a polar parallel to the axis of the Earth's rotation. Therefore, in the first case, two motors are used in the stand to automatically follow the sun; but in the second case it can be done with one motor. Orienting the solar panels by rotating them around the polar axis is essentially tilting the panels to the east or west. Because of this, during the turning the lower corners of the panels move downwards and upwards and the angle of rotation of the azimuth cannot exceed $180^{\circ}$ in the plane of the azimuth [3. To prevent the corners from touching the ground, the panel block should be lifted higher than the two-axle drive units, i.e. a significantly higher stanchion that increases the cost of the stand, lifts the panels into a stronger wind zone and makes maintenance of the devise more difficult. To overcome this deficiency, the design of the solar panel device [ 4 was created, in which the solar panels do not collapse as they rotate around the polar axis, they only change the angle of the zenith and azimuth. The height of the stanchion is therefore similar to that of a two-axle drive. Continuing the development of one-engine twin-drive systems, a model [5] has been developed, where the motor rotates in the horizontal (azimuth) plane only, while the panel motion trajectory in the zenith plane is formed by the slider-crank mechanism.

\section{Materials and methods}

The device under consideration is essentially a form of a zenith-azimuth stand, which uses a single motor to simplify the design of the stand. The stand orients a solar receiver, such as a solar panel, in an azimuthal plane. The orientation of the panel in the zenith plane is performed using a slider-crank mechanism. The device (Fig. 1) consists of a stanchion 6, on which the motor-gearbox 5 is mounted. Two bearings 2 are mounted on the end of the motor-gearbox 5 vertical secondary shaft 4 . 
In the bearings 2 , the horizontal shaft 3 is rotating. To the horizontal shaft 3 , solar panels 1 are placed, and between the bearings of the horizontal shaft 3 , one end of the crank 7 is fixed. At the other end of the crank 7 there is a hinge $\mathrm{A}$, which forms a moving joint at the upper end of the crank 7 with a slider 8. A similar joint of the pivot $C$ is at the other end of the slider 8 with a bracket 9 , the other end of which is permanently attached to the stanchion 6 .

Constructive length of the slider 8 is chosen so that the angle $A O C=90^{\circ}$ and the length of the bracket 9 to the angle $A O H=90^{\circ}-\varphi$, where: $\varphi$ - the latitude of the place of the use of the device. For Latvia $\varphi=57^{\circ}$ and the angle of the equinox $A O H=33^{\circ}$. Then, during the sun's equinox, at lunchtime the direction of the sunbeams will coincide with the axis of symmetry of the crank 7 . It has to be noted that for the stand to function properly, the axes of symmetry of the slider 8 , crank 7 , bracket 9 , and stanchion 6 should be in the same plane at lunchtime, facing the southern side of the stanchion to coincide with the direction of solar radiation during the lunchtime, when the equation of time is zero.

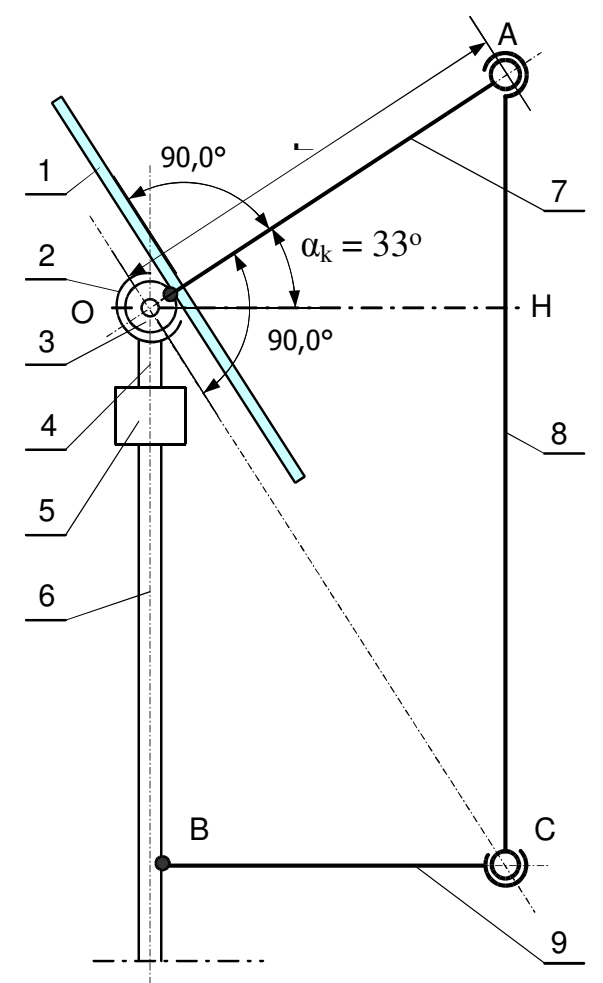

Fig. 1. Solar panel single motor twin drive equipment (side view at lunch in time of sun equinox): 1 - solar panels; 2 - bearings; 3 - horizontal shaft with solar panels; 4 - secondary shaft of motorgearbox; 5 - motor-gearbox; 6 - stanchion; 7 - crank; 8 - slider; 9 - bracket; OC - axis of symmetry of the cone; A; $\mathrm{C}$ - joints; $\mathrm{L}$ - length of the crank

The device works as follows. When the shaft 4 of the motor-gearbox 5 turns east or west, the crank 7 with the slider (connecting rod) 8 around the axis of symmetry $O C$ forms a rotating cone having a vertex $C$, a mould 8 and a base circumference $O A$ with a radius equal to the length of the crank 7. When turning the gearbox shaft 4 to the east or west, the crank angle $A O H$ relative to the horizontal plane $\mathrm{OH}$ decreases, and after turning by $90^{\circ}$, the crank axis of symmetry goes into the horizontal plane $O H$. The device control unit should make this turn evenly within $6 \mathrm{~h}$, but if from the morning to evening, then during $12 \mathrm{~h}$. The direction of the sunbeams should coincide with the direction of the axis of symmetry of the crank 7 ,

The trajectory of the crank 7 joint $\mathrm{A}$, that is, the angle ak of the crank height $\mathrm{AH}$ to the horizon (Fig.1) can be calculated graphically as a function of the azimuth turning angle, i.e. during one hour (Fig. 2). For that, from the cone base circle $90^{\circ}$ sector $B O A$ is released and divided into six parts by $15^{\circ}$, one hour at a time. The obtained points with the $O B$ parallels have transferred to the horizontal $O H$. Constructive $c$ length is chosen so that the angle $A O C=90^{\circ}$ and the length of the bracket $k-$ to the angle $A O H=90^{\circ}-\varphi$, where: $\varphi$ - the latitude of the place of the use of the device, e.g. $\varphi=57^{\circ}$ and 
the angle $A O H=33^{\circ}$. Then, during the sun's equinox at lunch time, the sun will cross the axis of the symmetry of the cliff (the direction of solar radiation will coincide with the axis of symmetry of the cliff). It has been noted that for the machine to function properly the axes of symmetry of the connecting rod, crank, bracket, and rack should be in the same plane at lunchtime, facing the southern side of the rack to coincide with the direction of solar radiation during the lunchtime, when the equation of time is zero.

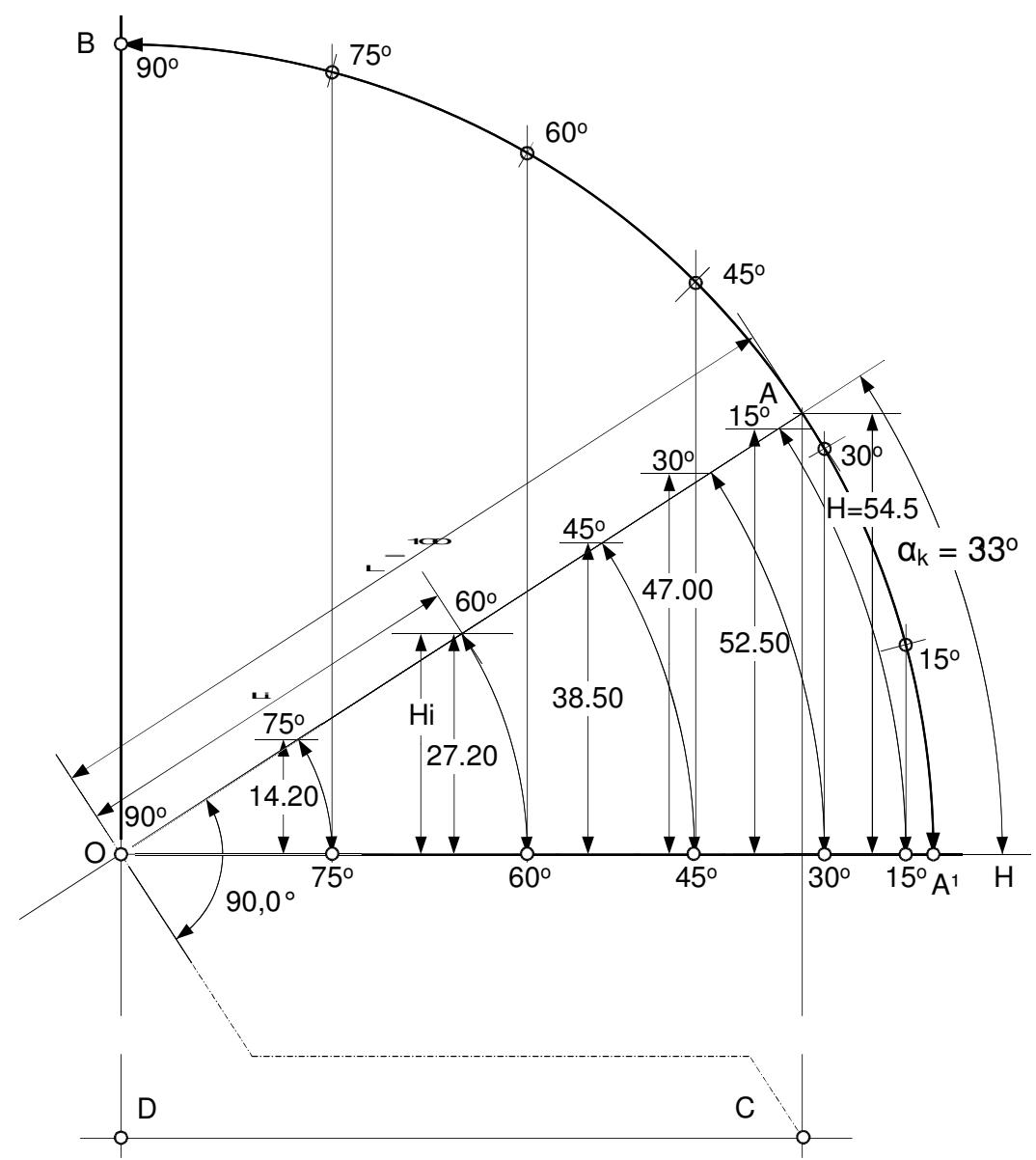

Fig. 2. Graphics for calculating solar panel trajectory of one engine two-axle drive unit

Using the expressions $(1 ; 4)$ in Fig. 2 , the angle of the crank height $\alpha_{k i}$ depends on the angle of the crank turn $A_{p}$. The data obtained are summarized in Tables 1 and 2, as well as graphically shown in Fig.3, together with the sun height angle curve.

Then, turning the horizontal line $O H$ counter-clockwise at the equinox angle, $\alpha_{k}=33^{\circ}$ we can calculate the corresponding height $\mathrm{Hi}$ for each crank turn by $15^{\circ}$ (height of the crank end above the horizon)

$$
\alpha_{k i}=\arcsin \frac{H_{i}}{L},
$$

where $\alpha_{k i}$ - angle of the crank height to the horizon;

$H_{i}$ - height of the crankshaf tend above the horizon;

$L$ - length of the crank (crankshaft).

Using Figure 2, we can make an equation for calculating the crank height angle in Excel.

The coordinates of the crank end trajectory can be expressed:

$$
H_{i}=L_{i} \cdot \sin \alpha_{k}
$$

and 


$$
L_{i}=L \cdot \cos A_{p},
$$

where $L_{i}-$ length of the crank in relation to the point $\mathrm{O}$;

$A_{p}$ - rotation angle of the crank relating to the horizon;

By combining both expressions, we obtain,

$$
H_{i}=L \cdot \cos A_{p} \cdot \sin \alpha_{k},
$$

which inserting in expression (1) we obtain

$$
\alpha_{k_{i}}=\arcsin \frac{L \cdot \cos A_{p} \cdot \sin \alpha_{k}}{L}
$$

or:

$$
\alpha_{k_{i}}=\arcsin \left(\cos A_{p} \cdot \sin \alpha_{k}\right)
$$

Using expressions $(1 ; 4)$ for in Fig. 2 given points, the angle of the crank height $\alpha_{k i}$ depending on the angle of the crank turn $A p$ has been calculated. The data obtained are summarized in Tables 1 and 2, as well as graphically shown in Fig. 3 together with the sun height angle characteristic curve.

In order to judge about the crank height angle to the horizon $\alpha_{k i}$ compliance to the angle of the sun's height, it is necessary to calculate the angle $\alpha$ of the altitude of the sun at the points (Fig. 2), considering the time and place of the stand used. From [6] it follows that the angle $\alpha$ of the altitude of the sun, depending on the hour of the sun (azimuth) angle $A$, is calculated as

$$
\sin \alpha=\sin \varphi \cdot \sin \delta+\cos \varphi \cdot \cos \delta \cdot \cos A,
$$

here

$$
A=(L S T \pm D) \cdot 15+180, \text { degrees }
$$

where $D$ - time difference between the clock and the sun, h;

$L S T$ - time of the local time zone.

$$
D=\left[\frac{P-R}{15}+E T\right], D=[(P-R) / 15+E T]
$$

where $P$ - latitude of the place (Latvia $\approx 24.3^{\circ}$ );

$R$ - degree of the time zone length, $\left(30^{\circ} ; 2 \mathrm{~h}\right)$;

$E^{T}$ - time leveling in the time equation $(\mathrm{ET} \approx-7 \mathrm{~min})[6]$.

In expression (7) $\mathrm{P}$ and $\mathrm{R}$ aregiven in degrees, ET and $\mathrm{D}$ - in hours.

Example of calculations: Calculate the sun height for Latvia, Ulbroka, on March 22 .

$P \approx 24.3^{\circ} ; R=30^{\circ}(2 \mathrm{~h})$; latitude $\varphi \approx 57^{\circ}$; solar declination angle $\delta=0^{\circ}$; time offset $E T \approx-7 \mathrm{~min}$.

$$
D=\left[\frac{P-R}{15}+E T\right]=\left[\frac{24.3-30}{15}+0.12\right]=-0.38 \mathrm{~h}-0.12 \mathrm{~h}=30 \mathrm{~min}
$$

This means that the sun's time lags behind the clock by 30 minutes and that the sun's lunchtime will come at 12:30 (after wintertime). The sun hour angle at 12:00.

$$
\begin{gathered}
A=(12-0.5) \cdot 15+180=352.5^{\circ} \\
\sin \alpha=\sin 57^{\circ} \cdot \sin 0^{\circ}+\cos 57^{\circ} \cdot \cos 0^{\circ} \cdot \cos \left(352.5^{\circ}\right) .
\end{gathered}
$$

As the sun declination angle $\delta=0^{\circ}, \sin 0=0, \cos 0=1$, then

$$
\sin \alpha=\cos 57^{\circ} \cdot \cos \left(352.5^{\circ}\right)=0.54 \text { and } \alpha=\arcsin \left(0.54^{\circ}\right)=32.7^{\circ}
$$


The calculated data on the sun angle using the equation (8) are summarized in Table 2 and graphically shown in Fig. 3, together with the crank height angles curve.

\section{Results and discussion}

Calculations of the crank height angle of the device under consideration against the horizon, depending on the crank turning angle $A p$, have been made. For producing calculation formulas a graphical image (Fig. 2) was used. The heights of the crank end $A$ against the horizon depending on the crank turning angles in every $15^{\circ}(1 \mathrm{~h})$ have been obtained. Applying these crank end heights to the length of the crank and calculating the sinus angles of these ratios, the height angles have been obtained as a function of the crank turning angles. Expressing point ( $\mathrm{Hi} ; \mathrm{Li})$ coordinates with angles of SIN and COS, an expression (3) have been obtained. Using this expression the calculation of the crank height angle as a function of the crank-turning angle without drawing can be done. An expression (4) has been created for calculating the crank height angle in Excel. The expressions $(1 ; 3 ; 4)$ have been used for the calculation of the crank height and for comparing the results, which are summarized in Table 1 and Table 2. As it can be seen from the tables, there are no significant differences in the magnitude of the data obtained.

Crank height angle $\alpha_{k i}$ depending on $A_{p}$ angle, using formulas $(1,4)$

Table 1

\begin{tabular}{|c|c|c|c|c|c|c|c|}
\hline Crank angle $\boldsymbol{A}_{\boldsymbol{p}}$, degrees & $\mathbf{0}$ & $\mathbf{1 5}$ & $\mathbf{3 0}$ & $\mathbf{4 5}$ & $\mathbf{6 0}$ & $\mathbf{7 5}$ & $\mathbf{9 0}$ \\
\hline Height of crank end, Hi & 54.500 & 52.500 & 47.000 & 38.500 & 27.200 & 14.200 & 0 \\
\hline Ratio, Hi / L & 0.545 & 0.525 & 0.470 & 0.385 & 0.272 & 0.142 & 0 \\
\hline Crank end height angle, $\alpha_{k i}(1)$ & 33.020 & 31.670 & 28.030 & 22.640 & 15.780 & 8.160 & 0 \\
\hline Crank end height angle, $\alpha_{k i}(4)$ & 33.020 & 31.760 & 28.160 & 22.660 & 15.810 & 8.100 & 0 \\
\hline
\end{tabular}

Data calculated by equations $(4,8)$

\begin{tabular}{|c|c|c|c|c|}
\hline $\begin{array}{c}\text { Deviation } \\
\text { angle, degrees }\end{array}$ & $\begin{array}{c}\text { Clock } \\
\text { time, } \mathbf{h}\end{array}$ & $\begin{array}{c}\text { Crank height } \\
\text { angle, degrees }\end{array}$ & $\begin{array}{c}\text { Sun height } \\
\text { angle, degrees }\end{array}$ & $\begin{array}{c}\text { Sun hour } \\
\text { angle, degrees }\end{array}$ \\
\hline 120 & 4 & -15.80 & -19.36 & 232.50 \\
\hline 90 & 6 & 0 & -4.08 & 262.50 \\
\hline 60 & 8 & 15.80 & 12.03 & 292.50 \\
\hline 30 & 10 & 28.14 & 25.60 & 322.50 \\
\hline 0 & 12 & 33.00 & 32.68 & 352.50 \\
\hline 30 & 14 & 28.14 & 30.21 & 382.50 \\
\hline 60 & 16 & 15.80 & 19.36 & 412.50 \\
\hline 90 & 18 & 0 & 4.08 & 442.50 \\
\hline 120 & 20 & -15.80 & -12.03 & 472.50 \\
\hline
\end{tabular}

Note: Table 2 shows the results for days in pairs only in order to reduce the amount of the data shown.

In order to judge about the angle of the crank height to the horizon compliance with the angle of the sun's height, the methodology given in [4] was applied. The sun height angle, depending on the angle of the sun hours (azimuth) has been calculated. The data obtained are summarized in Table 2, together with the crank height angle. Fig. 3 shows the graphical data. In Fig. 3a we can see that the solar panels are the sun oriented at 12:00 clock time and the sun is behind $(-0.5 \mathrm{~h})$. By turning the device to 7.5 degrees west, we get a complete coincidence of the crank and the sun height angles (Fig. 3b). The curves coincide, if $P=12: 30$, which was used in the calculations by formula (7).

Fig. 4 demonstrates the characteristic adjustment curves obtained by periodically rotating them to the shaft 3 (Fig. 1) to $\approx \pm 20$ degrees from the solar equinox time to compensate for changes in the solar declination during the year.

As we can see, in all cases the solar panels have been turned about $0.5 \mathrm{~h}$ west. The coincidence of the crank (normal surface of the solar panels) and the angle of the sun could then be similar to that of the solar equinox and that would be good enough. 

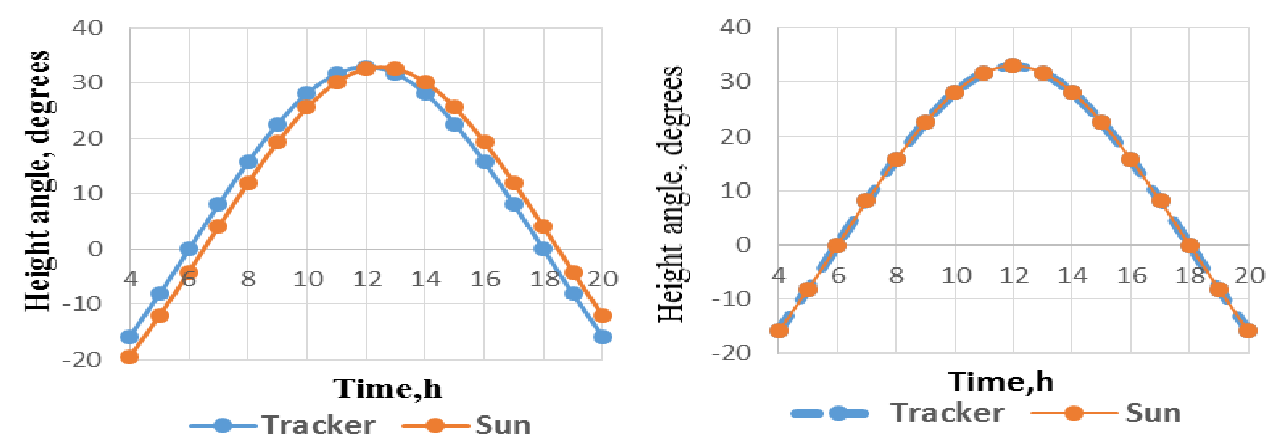

Fig. 3. Crank of device and sun height angles as function of time (crank turn angle):

Fig. $3 a$ - situation at 12:00 clock time; Fig. $3 b$ - situation after turning panels by 7.5 degrees west

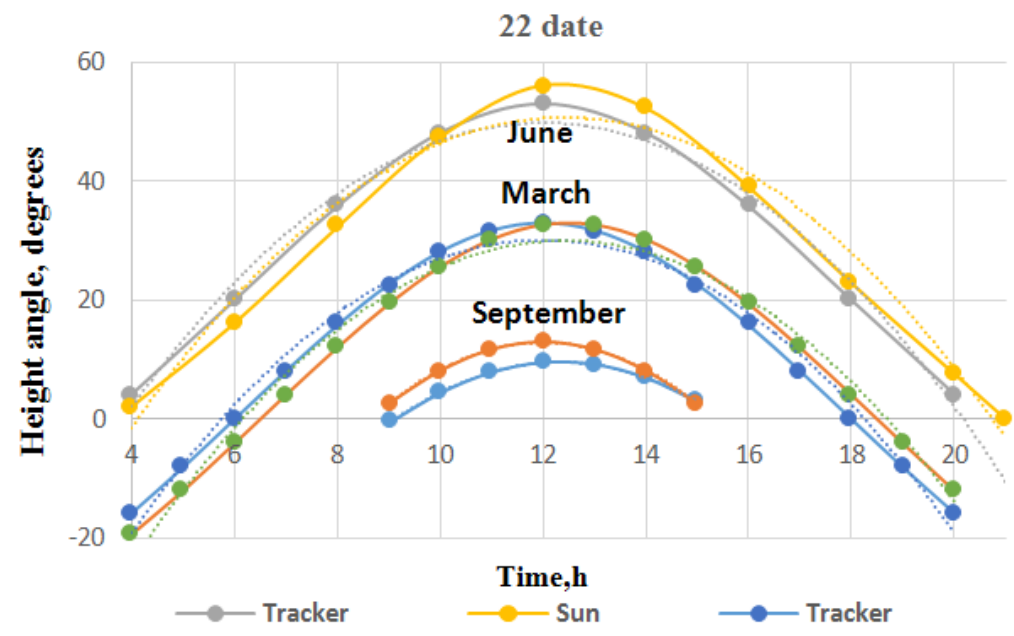

Fig. 4. Adjustment curves by changing panel angle to crank due to sun declination

\section{Conclusions}

1. To obtain formulas for the theoretical calculation of the crank turning angle, the graphical method was used.

2. The angle between the crank of the device and horizon, depending on the crank turning angle, was calculated.

3. Using Excel programme, the calculated values of the crank angles referring to the horizon with the sun angles were compared and high coincidence was obtained at the level up to $92 \%$.

4. It is possible to use the worked out method for substantiation of the construction, as the difference between the values calculated and experimentally obtained is insignificant.

\section{References}

[1] Dual-axis solar tracker for 4 panels ST44M2V4P [online] [06.04.2018]. Available at: http://wwwsolar-motors.com (www.solar-motors.com).

[2] New Approach on Development a Dual Axis Solar Tracking Prototype. [online] [06.04.2018] Wireless Engineering and Technology 07(01):1-11, DOI: 10.4236/wet.2016.71001

[3] Deepthi S., Ponni A., Ranjitha R., etc. Comparison of Efficiencies of Single-AxisTracking System and Dual-Axis TrackingSystem with Fixed Mount, International Journal of Engineering Science and Innovative Technology Volume 2, Issue 2, March 2013(IJESIT). [online] [06.04.2019]. Available at: http://www.ijesit.com/Volume\%202/Issue\%202/IJESIT201302_66.pdf

[4] Putāns H., Ziemelis I., Pelēce I., Ivanovs S., Sṇegovs A., Kanceviča L.. Saules bateriju saulei sekošanas ierīce (The sun tracking device for solar batteries). Patents, LV 15245 B, 2016. (In Latvian).

[5] Paulek V., Libra M. Solar energy. CUP, 2006, 153 p.

[6] Харкнесс Е., Мехта М. Регулирование солнечной радиации в зданиях (Rregulation of solar radiation in premises), Москва Стройиздат 1984. 177 стр. (In Russian). 